\title{
On progression in meditation: habit, curiosity, and identity
}

\author{
Ho Manh Tung \\ Ritsumeikan Asia Pacific University \\ Beppu, Oita, Japan
}

September 27, 2020

In this essay, I want to reflect on what it means to make progress in meditation. There are plenty of pieces of good advice in Sam Harris's book, Waking $U p^{1}$ and his app, which I used to keep track of my formal sitting meditation. In one of the lessons in the app, Harris also talks about progress in meditation. I will summarize some of it as but also add my thoughts on the subject.

\section{Formal meditation becomes a habit}

1) Formal sitting meditation becomes a part of your daily routine. You find it easier to sit down for 5-10 minutes a day, paying attention to the breath, sensations, moods, emotions, and thoughts in mind. This is the first sight of making progress in meditation.

\section{Mindfulness becomes more of a mode of being}

2) Informal mini-meditations during the day start to become more regular. You find your day getting more punctuated by brief moments of open and clear awareness about what you are doing, thinking, feeling. You are more mindful and reflective when getting up from your desk, when switching tasks, when going online, when beginning to work, when taking a walk, etc. 


\section{Experiencing the world with a little more empathy, intensity, and tenderness}

3) Your day becomes more punctuated by moments of tenderness. I am raising two small children, so this aspect is more salient to me. I always tend to feel a pang of emotions when I realize they stop doing some children things, or when my wife points out that I have missed some cute things they did. The more mindful of the finite nature of life, the more intensity and tenderness one feels towards people and things.

4) It is easier to empathize and even identify with the pain and joy of other people. It is not something that you can force. At some point in your meditative journey, you suddenly realize it is easier for your mind to register the pain and joy of other people more.

\section{Clear viewing of what the mind's doing}

5) Starting to realize how often you are carried away by thoughts. The more concentration you build up in mind, the more you realize how easy it is to get lost in thoughts without knowing it. It is kind of a paradox. The whole exercise of mindfulness is to realize that

you have been lost in thoughts, clearly seeing the thoughts and their disappearance in your awareness.

6) Starting to realize the subtle reactions you have toward everything. The more mindfulness you build up, the more you realize that it seems you have a subtle judgment about everything you encounter. There seems to be an automatic mechanism in the mind that assigns values very quickly to everything, even your own thoughts, and feelings: "I like it," "I don’t like it," "I don't care."

7) Starting to see clearly the interaction among thoughts, moods, and emotions. One of the things you'll learn beginning to meditate is to be aware of your moods and bodily sensations 
for every formal meditation session. Once you have more mindfulness, it becomes clearer how your moods and emotions drive your thoughts, and vice versa. I know for certain now if my body is tired, my mind is more likely to have frustrations, which can make me more likely in dwelling in negative thoughts; if I feel good in my body, my mind is more likely to be excited and inspired, which makes it easier to be carried away by thoughts.

8) Starting to quietly acknowledge both the presence as well as the absence of something in your mind. The most salient example is your emotion. As you become more mindful, you will notice you have anger or anxiety or excitement in your mind, and how those emotions drive your thoughts and actions. At the same time, you will become more mindful of the absence of such emotional states. The same process goes for your thoughts, bodily sensations, etc.

9) Starting to realize some of your subtle emotional reactions in a social situation. I always find the social situation to be trickier. There seems to be always an urge to signal certain aspects of yourself. There seems to be always an urge to adjust what you are saying to the subtle emotional expressions other people making.

10) Starting to have more buffers in between stimuli and responses. This is a direct result of seeing more clearly what the mind is doing in each moment. By definition, when you are not caught up in the moment, when you are not carried away, you have more buffers in between stimuli and responses. You can choose your response better.

\section{More curiosity and serendipity}

11) Starting to have more curiosity about yourself and others. By seeing what the mind does more clearly, you can dampen your default, automatic reactions to things and people more. As your mind is more open, it becomes more curious about many more things in life ${ }^{2}$. Daniel 
Goleman and Richard Davidson show that the brain of high-level meditator is correlated with frequent exertion of EEG gamma brainwave frequency, which is associated with the mental state of discovery ${ }^{3}$. Brewer et al. (2011) argue in the context of quitting additive behavior such as smoking, by opening your mind with mindfulness, meditators replace smoking with the feeling of curiosity, which is inherently pleasurable itself, in response to the stimuli of craving a cigarette $^{4}$.

12) More serendipitous moments and ideas start to appear. As your mind becomes more open and understanding, it is easier for you to register ideas and see how they can fit your core values. In other words, your mindsponge is getting better at filtering ideas and values ${ }^{5,6}$.

\section{Identities become less tangible and unnecessary suffering disappears}

13) Seeing everything more and more as interdependent and interconnected. Gaining more understanding in yourself how thoughts, emotions, behaviors, sensations are doing and relating to, in each moment, it becomes easier to extend that to other people and things.

14) Seeing more and more the layers of concepts and conditionings that shape your perception of the world. This point is directly related to the previous points on interdependence and interconnectedness. Once you think about how each thought is shaped by many concepts, and each concept is dependent in a web of other concepts, it is hard to not to see meditation is like a knife that helps you peel away layers and layers of concepts that shape your perception of the world. In the Waking Up apps, Sam Harris points out we can lose the grip of the concept of a hand by simply identify with the raw sensations of temperature, humidity, pressure, tingling, touch. Having a laser-like focus on the raw sensations, the concept of the body can fades away. 
15) All the knowledge about what the mind does make all narratives about yourself and others less tangible. I see this is as a direct result of clearly seeing the mind: becoming less attached to what you identify with, in yourself and others, is possible. It is possible to take on a certain identity until it is useful. One can be a cautious person or a reckless one if it is useful. One can be a brave person or a shy one. One can be assertive, or one can be quiet. The point is more clear-seeing translates into more understanding, which translates into more choices. I see it in myself a lot that I sometimes used self-identities and perceptions of others as excuses not to do something.

15) Knowing how to end the unnecessary kinds of suffering. Too much of our day to day suffering is caused by our attachment to some identities, or by our getting lost in thoughts ${ }^{7}$. It is clear that losing the grip of identities and being able to take a step back from your own thoughts can be a very direct way of ending the unnecessary kinds of suffering. Correctly being mindful, you will naturally learn that you can be as angry as it is useful, as anxious as it is useful. Suffering or problems are, in and of themselves, arguably important to maintaining a productive and reasonable life. But perpetuating suffering unnecessarily is not a wise strategy.

16) Killing the Buddha. In this Sam Harris' blogpost $^{8}$, he wrote the ninth-century Buddhist master Lin Chi is supposed to have said, "If you meet the Buddha on the road, kill him." What Harris is trying to drive at citing the Buddhist master is that there is a problem of identifying Buddhism as a religion and makes a case that to be a true Buddhist, one must eventually letting go of this identity. He wrote:

"In many respects, Buddhism is very much like science. One starts with the hypothesis that using attention in the prescribed way (meditation), and engaging in or avoiding certain behaviors (ethics), will bear the promised result (wisdom and psychological well-being). This spirit of 
empiricism animates Buddhism to a unique degree. For this reason, the methodology of Buddhism, if shorn of its religious encumbrances, could be one of our greatest resources as we struggle to develop our scientific understanding of human subjectivity."

In the context of mediation, the ultimate sign of progress, to me, is the letting go of all attachments and identities: the killing of the ego. The ego is very tricky. Sometimes, it can even turn Buddha into a part of it. Much of the problems in the world is caused by our egos masquerading as some kinds of virtues ${ }^{9}$, thus, clearly seeing what is taking place in the mind and how it drives our behaviors, thoughts, and emotions is the first step towards making yourself a better person and making the world a better place.

\section{Further resources:}

Try one-month free of Sam Harris' Waking Up app here:

https://dynamic.wakingup.com/redeemMonth/1448fb

\section{References}

1. Harris, S. (2014). Waking up: A guide to spirituality without religion. NY: Simon and Schuster.

2. Brewer, J. A., Davis, J. H., \& Goldstein, J. (2013). Why is it so hard to pay attention, or is it? Mindfulness, the factors of awakening, and reward-based learning. Mindfulness, 4(1), 75-80.

3. Goleman, D., \& Davidson, R. J. (2017). Altered traits: Science reveals how meditation changes your mind, brain, and body. London: Penguin.

4. Brewer, J. A., Mallik, S., Babuscio, T. A., Nich, C., Johnson, H. E., Deleone, C. M., ... \& Carroll, K. M. (2011). Mindfulness training for smoking cessation: results from a randomized controlled trial. Drug and Alcohol Dependence, 119(1-2), 72-80. 
5. Killingsworth, M. A., \& Gilbert, D. T. (2010). A wandering mind is an unhappy mind. Science, 330(6006), 932-932.

6. Napier, N.K. \& Vuong, Q.H. (2013). Serendipity as a strategic advantage?. In Wilkinson (ed) Strategic Management in the 21st Century (pp. 175-199). Westport, CT: Praeger/ABC-Clio. DOI: $\underline{10.13140 / 2.1 .3311 .952 .}$

7. Vuong, Q. H. \& Napier, N. K. (2014) Making creativity: the value of multiple filters in the innovation process. Int $J$ Transitions and Innovation Systems, 3(4), 294-327. DOI: 10.1504/IJTIS.2014.068306.

8. Harris. S. (2006). Killing the Buddha. Retrieved from https://samharris.org/killing-the-buddha/ (accessed: September 25, 2020).

9. Vuong, Q. H., Ho, M. T., Nguyen, H. K. T., et al. (2020). On how religions could accidentally incite lies and violence: Folktales as a cultural transmitter. Palgrave Communications, 6(1), 82. 